\section{Pius S \\ Bello M \\ Mava Y \\ Djossi S \\ Ambe JP}

\title{
Prevalence of exchange blood transfusion in severe hyper- bilirubinaemia and outcome at the University of Maiduguri Teaching Hospital Maiduguri, North- eastern Nigeria
}

DOI:http://dx.doi.org/10.4314/njp.v44i2.2

Accepted: 9th November 2015

Pius S (『)

Bello M, Djossi S, Ambe JP

Dapartment of Paediatrics,

University of Maiduguri Teaching

Hospital Maiduguri, Nigeria

Email:

simonpius2000@yahoo.co.uk

Mava Y

Department of Paediatrics,

Bingham University Teaching

Hospital, Jos, Nigeria

\begin{abstract}
Background: Exchange blood transfusion (EBT) is carried out for the treatment of conditions presenting with severe hyperbilirubinaemia and anaemia, such as $\mathrm{ABO}$ incompatibility, sepsis, prematurity and birth trauma among others. While it is fast being abandoned as treatment modality for severe neonatal jaundice in the resource rich countries, it is still a backbone of treatment for severe neonatal jaundice in resource limited settings. Since such a study has not been done in this centre before now, we decided to study the positive effects of exchange blood transfusion in Maiduguri, North-Eastern Nigeria.

Objective: To determine the prevalence, indications and outcome of exchange blood transfusion at the Special Care Baby Unit of the University of Maiduguri Teaching Hospital, Maiduguri.

Methods: This is a retrospective study which examined the records of babies admitted into the SCBU between $1^{\text {st }}$ January to December $31^{\text {st }} 2014$ with the aim of determining the prevalence of EBT at the Special Care Baby Unit (SCBU) of the University of Maiduguri Teaching Hospital (UMTH), Maiduguri.The indications for EBT and mortality among babies who had EBT are also highlighted.

Results: A total of 639 babies with gestational ages between 30 weeks to 42 weeks were admitted into the Special Care Baby Unit (SCBU) over the period of
\end{abstract}

12 months. Of this number, 64 (10\%) had neonatal jaundice. Thirty $(46.9 \%)$ of the 64 neonates with NNJ had EBT, their preEBT, serum bilirubin (SB) level ranged from $15 \mathrm{mg} / \mathrm{dL}$ to $28.5 \mathrm{mg}$ / $\mathrm{dL}$ with mean of $21.5 \pm 13.0 \mathrm{mg} / \mathrm{dL}$, while the post EBT SB ranged from $3.0 \mathrm{mg} / \mathrm{dL}$ to $11.3 \mathrm{mg} / \mathrm{dl}$ with mean of $7.2 \pm 8.3 \mathrm{mg} / \mathrm{dL}$. The aetiological risk factors of severe hyperbilirubinaemia in the newborns who had EBT include ABO incompatibility $17(56.7 \%)$, sepsis 12 $(40.0 \%)$, prematurity $10(33.3 \%)$. Out of 30 neonates had EBT, 25 $(83.3 \%)$ survived and were discharged, 5(16.7\%) died and 3 with bilirubin encephalopathy, 1 with severe perinatal asphyxia with hypoxic ischaemic encephalopathy stage II while the fifth death was a preterm extreme low birth weight. Conclusion: In our setting, the prevalence of exchange blood transfusion is high and this is because a large number of our patients had severe NNJ at presentation, some with bilirubin encephalopathy. Exchange blood transfusion remains one of the most reliable and effective treatment modality to prevent bilirubin encephalopathy especially in settings where babies are brought late to health facility. Effort at health education of the population at risk, especially pregnant women on early recognition and referral to appropriate health facility for prompt treatment to prevent severe NNJ and bilirubin encephalopathy is highlighted. 


\section{Introduction}

Exchange blood transfusion (EBT) is a procedure done for removal of antibodies coated red blood cells and/or products of haemolysis in a variety of neonatal conditions which present with severe unconjugated hyperbilirubinaemiadue such as prematurity, rhesus isoimmunization, ABO incompatibility. or non immune haemolytic anaemia as in Glucose 6 phosphate dehydrogenase (G6PD) deficiency. ${ }^{1}$ It is also done in conditions like severe neonatal sepsis for there moval of bacterial toxins, severe respiratory distress syndrome, perinatal asphyxia, disseminated intravascular coagulopathy and poisoning among others. ${ }^{2-6}$

EBT involves removing aliquots of patient's blood and replacing it with donors. ${ }^{7}$ The prevalence of EBT reported from Nigeria by Owa and Ogunlesi ${ }^{8}$ Onyearugha et $\mathrm{al}^{9}$ were high while reports from developed countries showed that EBT is now rarely carried out especially for unconjugated hyperbilirubinaemia because of the efficacy of phototherapy in reducing plasma bilirubin concentrationsthus preventing cerebral damage. ${ }^{4,5}$ Also, another report from South-western Nigeria showed that more than $5 \%$ of neonates in their unit had EBT. ${ }^{7} \mathrm{Re}$ ports from the developed world has demonstrated the efficiency of phototherapy in the treatment of neonatal jaundice, thereby abandoning EBT, but such cannot be said for developing countries like Nigeria where patients presents very late to health facilities, often with severe neonatal jaundice, some with bilirubin encephalopathy. Indications for exchange blood transfusion for healthy term babies is done at TSB level of $>25 \mathrm{mg} / \mathrm{dl}$, however; clinical signs of bilirubin encephalopathy is also an indication for EBT whatever the TSB level. In sick newborns, intervention is required at lower TSB levels mentioned above. For the preterm, the rule of 10 is applied where by the birth weight is multiplied by 10 and the figure obtained is the EBT TSB level. Besides the TSB level, there are many known factors like higher permeability of blood brain barrier, hypoxia, hypercarbia, sepsis and various unknown factors for bilirubin toxicity which will require EBT to be done at lower TSB levels. ${ }^{7}$ 10,11

EBT especially double volume exchange had succeeded in reducing mortality from $35-40 \%$ pre EBTera to less than $20 \%$ and the risk of neurological sequelle from $90 \%$ to $30 \%{ }^{12,13}$

This beneficial live saving method of treatment of neonatal conditions has its complications including air embolism, metabolic derangements, transmission of infections and gut perforation among others ${ }^{1,14}$ Mortality of between $10 \%-17.5 \%$ has been recorded. ${ }^{8,12,15,16} \mathrm{EBT}$ is now rarely carried out in developed countries because of the availability of efficient phototherapy units coupled with early detection and reporting to health facility with prompt monitoring of the patients. ${ }^{9}$ Shortage of phototherapy machines in many centres, interrupted power supply and late presentation of patients to hospitals, makes it almost impossible to rely mainly on phototherapy as treatment modality in severe neonatal jaundice (NNJ) in the developing countries, such as Nigeria.
There were no documentations on EBT, its indications and outcome in Maiduguri and the North-Eastern part of Nigeria. Therefore we decided to retrospectively review the prevalence, indications and outcome of exchange blood transfusion at the Special Care Baby Unit (SCBU), University of Maiduguri Teaching Hospital, Maiduguri; North-Eastern Nigeria.

\section{Subjets and methods}

This was a retrospective study conducted at the Special Care baby Unit (SCBU) of the University of Maiduguri Teaching Hospital, Maiduguri, Borno state Nigeria. The SCBU admits newborns as referrals from other hospitals in addition to newborns delivered at the centre and admitted for various illnesses including neonatal jaundice. The SCBU is run by two consultants, a senior registrar, two registrars, two interns and three nursing staffs on every shift.

Data were extracted from the folders of all babies who had exchange blood transfusion for their conditions at the SCBU, thus constituting the subjects for the study. Informations extracted includes, patients age, sex, gestational age, maternal/paternal and babies blood group, Rhesus typing, weight as at admission and relevant investigations done include bilirubin estimation both total and conjugated fractions, packed cell volume, complete blood count, blood culture among others. The treatment administered to the patients such as phototherapy, EBT, antibiotics, intravenous fluid and intranasal oxygen among others and outcome of the patients such as whether patients got well and discharged home, left against medical advice or died were all documented. Patients who had transfusion or EBT elsewhere before presenting to our facility were excluded because that might interfere with the actual picture of the conditions due to interventions received before referral.

At presentation, these patients were placed on phototherapy while awaiting for their serum bilirubin results which was obtained from the laboratory within

1-2hours. Exchange blood transfusion was carried out on all patients who had high unconjugated hyperbilirubinaemia. Exchange blood transfusion was performed at serum bilirubin of $20 \mathrm{mg} / \mathrm{dl}$ or below this level if the conjugated fraction was less than $20 \%$ of total bilirubin level in term newborn, while in preterm's exchange blood transfusion was performed at level 10$12 \mathrm{mg} / \mathrm{dl}$ or less in the presence of other factors such as septicaemia, severe birth asphyxia and other comorbidities like disseminated intravascular coagulation (DIC), kernicterus, anaemia. ${ }^{17}$ Double volume $(160 \mathrm{ml} /$ $\mathrm{kg}$ ) of fresh citrate phosphate dextrose anticoagulant containing whole blood which was usually less than 24hours, however blood less than three days was also accepted for EBT in severe hyperbilirubinaemia, while a single volume $(80 \mathrm{ml} / \mathrm{kg})$ for treatment of severe anaemia and at aliquots of $5 \mathrm{ml} / \mathrm{kg}$ according to the standard protocol. $^{11,15,17}$

The patients had EBT on admission using the pull and 
push method through an umbilical catheter and lasted between $45-60$ minutes and $1 \mathrm{ml}$ of $10 \%$ calcium gluconate was administered after every $100 \mathrm{ml}$ of blood exchanged in accordance with standard protocol. ${ }^{17}$ PreEBT and post-EBT serum bilirubin were estimated which was used to compare the efficacy of the procedure. In some patient, EBT had to done twice or more before the desired reduction in the serum bilirubin was obtained.

\section{Statistical Analysis}

Statistical analysis was done by SPSS version 16, Illinois, Chicago USA. Simple descriptive tests were done, Student t-test to compare mean values between groups and Chi squared test to test association between categorical variables. A P-value $<0.05$ was considered significant. Tables were appropriately used for illustration.

\section{Results}

A total of 639 babies whose gestational age at birth ranged from 30 weeks to 42 weeks with various conditions were admitted into the Special Care Baby Unit (SCBU) during the study period, between $1^{\text {st }}$ January and $31^{\text {st }}$ December 2014. Of this number, $64(14.6 \%)$ had neonatal jaundice. Thirty $(46.9 \%)$ of the 64 neonates had severe hyperbilirubinaemia and required EBT. All the 30 neonates had EBT carried out at admission because they had severe hyperbilirunaemia on arrival at our facility and their pre-EBT serum bilirubin (SB) level ranged from $15 \mathrm{mg} / \mathrm{dL}$ to $28.5 \mathrm{mg} / \mathrm{dL}$ with mean of 21.5 $\pm 13.0 \mathrm{mg} / \mathrm{dL}$, while the post EBT SB ranged from $3.0 \mathrm{mg} / \mathrm{dL}$ to $11.3 \mathrm{mg} / \mathrm{dl}$ with mean of $7.2 \pm 8.3 \mathrm{mg} / \mathrm{dL}$. Eight patients that had a second session of EBT before their serum bilirubin levels dropped down to acceptable level, were from the out borns, the remaining 22 $(73.1 \%)$ did well on one first session of EBT and follow up phototherapy. Taking a leap from the results following EBT procedures, there was rapid and significant reduction in the serum bilirubin levels of these patients who had exchange blood transfusion therapy.

There were 22 males and 8 females with a M: F of 2.8:1. Ten of them were preterm's, 19 term babies and one was post term baby. Nineteen of the babies $(63.3 \%)$ ) were of normal birth weight, $10(33.3 \%)$ has LBW, all were preterm infants with one macrosomic infant. The weight distribution of the babies range from $800 \mathrm{gm}-4300 \mathrm{gm}$ with the mean weight of $2550 \pm 750 \mathrm{gm}$.

Table I. Shows the socio demographic characteristics of the study population,

Forteen $(47.0 \%)$ of the mother were of younger age group $(<16-<25 y r s)$. ABO in compatibility was responsible for more than half of the causes of neonatal jaundice.

\begin{tabular}{lll}
\hline \multicolumn{2}{l}{ Table 1: Socio-demographic characteristics of the 64 patients } \\
with jaundice & & \\
Characteristics & Frequency & Percentage \\
\hline Mothers age & & \\
$<16$ & 02 & 06.7 \\
$16-25$ & 12 & 40.0 \\
26-35 & 14 & 46.6 \\
>35 & 02 & 06.7 \\
Total & 30 & 100.0 \\
Maternal blood group & & \\
A+ & 10 & 33.3 \\
B- & 03 & 10.0 \\
O+ & 17 & 56.7 \\
Total & 30 & 100.0 \\
Patients blood group & & \\
A+ & 07 & 23.3 \\
B+ & 10 & 33.3 \\
O+ & 13 & 43.4 \\
Total & 30 & 100.0 \\
Paternal blood group $*$ & & \\
A+ & 07 & 23.3 \\
B+ & 08 & 26.7 \\
O+ & 02 & 6.7 \\
Total & 17 & 56.7 \\
Patients weight (gm) & & \\
800-1499 & 06 & 20.0 \\
1500-2499 & 04 & 13.3 \\
2500-3999 & 19 & 63.3 \\
>4000 & 01 & 03.4 \\
Total & 30 & 100.0 \\
Age at admission & & \\
$<7$ days & 7 & 23.3 \\
7-28days & 23 & 76.7 \\
Inborn of delivery & & 33.3 \\
Outborn & & \\
\hline
\end{tabular}

*The paternal blood group was only available in 17 cases

Table 2: Shows the clinical features of patients who had EBT. Twenty three $(76.7 \% 0)$ of the patients had pyrexia and of these patients all the 30 (100.0) had jaundice. Severe anaemia accounted for 16 patients $(53.3 \%)$ and most of these patients had more than one symptom at the time of admission.

\begin{tabular}{|c|c|c|}
\hline Clinical features & Frequency & Percentage \\
\hline \multicolumn{3}{|l|}{ Temperature } \\
\hline$>37.5 \quad$ (hyperthermia) & 23 & 76.7 \\
\hline 36.5-37.5(normothermia) & 03 & 10.0 \\
\hline$<36.5 \quad$ (hypothermia) & 04 & 13.3 \\
\hline Fever & 20 & 66.7 \\
\hline Refusal to feed & 18 & 60.0 \\
\hline Anaemia* & 16 & 53.3 \\
\hline Difficulty in breathing & 11 & 36.7 \\
\hline Cycling movement & 03 & 10.0 \\
\hline Bleeding & 03 & 10.0 \\
\hline Convulsion & 03 & 10.0 \\
\hline
\end{tabular}

*Severe anaemia, defined by $\mathrm{PCV}<35 \%$, a patient had more than one symptoms 
Table 3 Shows the aetiological risk factors of severe hyperbilirubinaemia, $17(56.7 \%)$ of the children, the jaundice was due ABO incompatibility, 12 (40\%) patients had blood culture proven sepsis. Prematurity was responsible for severe hyperbilirubinaemia in 10 $(33.3 \%)$.

\begin{tabular}{lll}
\hline \multicolumn{3}{l}{ Table 3: Risk factor of neonatal jaundice } \\
Risk factor & Number of patients & Percentage \\
\hline ABO & 17 & 56.7 \\
Sepsis & 12 & 40.0 \\
Prematurity & 10 & 33.3 \\
Asphyxia & 08 & 26.7 \\
Birth trauma & 06 & 20.0 \\
Unknown & 12 & 40.0 \\
\hline
\end{tabular}

*some patients had more than one problem

Table 4 shows the outcome of the EBT in the 30 neonates. $25(83.3 \%)$ of the patients recovered and were discharged and subsequently seen in the neonatal clinic follow-up up to the age of three months before some were lost to follow up.

\begin{tabular}{lll}
\hline \multicolumn{3}{l}{ Table 4: Outcome of the patients Following EBT } \\
Outcome & Frequency & Percentage \\
\hline Discharge & 25 & 83.3 \\
Died & 05 & 16.7 \\
Total & 30 & 100.0 \\
\hline
\end{tabular}

While $5(16.7 \%)$ died, of these three had kernicterus even before EBT was done all died within $24 \mathrm{hrs}$ after EBT, while the one with severe perinatal asphyxia died three days after EBT, and the fifth death was preterm extreme low birth weight who weigh $800 \mathrm{gm}$ at birth and presented within $24 \mathrm{hrs}$ to hospital, had EBT successfully but died on the $7^{\text {th }}$ day of admission $(\chi=0.32, P>0.05)$

\section{Discussion}

The prevalence of exchange blood transfusion (EBT) in this study was $46.9 \%$, this was higher than $5.8 \%$ reported by Owa et $\mathrm{al}^{8}$ from South-western Nigeria and the report by Mills, ${ }^{18}$ however, this may not be the whole picture of the burden as their study was the proportion of the whole admission. The finding in our study was also much higher than the report by Onyearugha et al, Ibekwe et al from Abakaliki, South-eastern Nigeria, who reported the EBT rate of $20.8 \%, 17 \%$ respectively in their survey, ${ }^{8,9,11,19}$ the reason for the differences may be that while in our study it was over one year and majority of the newborns were delivered at home or poorly equipped health facility and we only saw them when they presented with severe neonatal jaundice.

Also mothers of these patients were not much aware of the severity and the effect of neonatal jaundice on their babies, in their survey and they had a large number of patients with neonatal jaundice managed in their NICU. Secondly the mothers of the patients studied at
Abakaliki, might have been more enlightened on the dangerous effect of neonatal hyperbilirunaemia to their newborns and so presented in large number and early enough to the health facility where they received intervention.

The prevalence rate of EBT of $46.9 \%$ was higher than the finding reported from Canada with an EBT rate of $22.1 \%$ for NNJ and reason being attributed to the practice of early discharge of patients home usually within 24hours only to be re-admitted with severe NNJ jaundice warranting EBT. ${ }^{20}$ The effectiveness of exchange blood transfusion therapy as the most efficient modality of management of severe hyperbilirubinaemia in our setting like in other under resources setting, was clear judging from the differences between the pre-EBT serum bilirubin results and the post-EBT results which showed a significant reduction in the serum bilirubin level. ${ }^{9,} 11$ The common presentation observed among the children that had EBT for severe hyperbilirubinaemia was jaundice $30(100 \%)$, others include fever/pyrexia in about $20(66.7 \%)$ patients, refusal to feed in $18(60.0 \%)$ patients, severe anaemia in $16(53.3 \%)$ patients, the reason might be because in our patient Sepsis was second only to $\mathrm{ABO}$ incompatibility as possible cause of NNJ which also has been reported in other studies. ${ }^{21,22}$ Other manifestations observed in the children that had EBT included opisthotonus, bleeding and convulsion.

This study has demonstrated that $\mathrm{ABO}$ incompatibility $17(56.7 \%)$ accounted for aetiological risk factor of neonatal jaundice, this was followed by sepsis which was responsible for $12(40.0 \%)$ and prematurity $10(33.3 \%)$ as the leading aetiological risk factors for neonatal jaundice, and sepsis was more among the outborns, while prematurity were mainly in the inborns, this finding was in keeping with the report from South-eastern Nigeria, ${ }^{22}$ and South-East Asia. ${ }^{23}$

Aetiological risk factors were not found in 12 (40.0\%), this has also been reported earlier and since we are incapable do screening for other causes of NNJ like G6PD deficiency, we cannot say with certainty it had not contributed to the jaundice observed in this study. ${ }^{9}$ The outcome of EBT in these neonates was categorized into the proportion of $25(83.2 \%)$ responded well to the treatment and was discharged home and subsequently seen at follow-up in neonatal clinic up to the age of three months with no apparent problems. In this study mortality rate of $5(16.7 \%)$ was obviously high compare to the $0.34 \%-10 \%$ finding reported earlier from South-western part of Nigeria, ${ }^{8}$ and those from outside Nigeria $0.5 \%$ in USA, $0.75 \%$ in Greece and $1.5 \%$ from Iran, ${ }^{24}$ the possible explanation may be that our patient were mostly referred from outside and often presented late to the hospital and another plausible explanation include rampant interruption of power supply leading to ineffective phototherapy regiment, these was acknowledged by others, ${ }^{9,11}$ however it was similar to the finding reported from Abakaliki South-eastern Nigeria, ${ }^{11}$ who found mortality rate $17.5 \%$ and that from Canada mortality rate of $20.8 \%$ for obvious reasons mentioned earlier. ${ }^{20}$

Just like in other low resources setting, payment for 
medical care was usually out of pocket expenses, ${ }^{9}$ such a lack of fund lead to delay presentation to hospital. As such $66.7 \%$ of the patients presented after first week of life with severe jaundice, we infer it might be attributed the delay to lack of finance. Some researchers have demonstrated that measuring the serum bilirubin level within the first 24hours of life is said to be helpful in predicting which infants will experience severe hyperbilirubinaemia with hope of early detection and monitoring of the condition. ${ }^{24}$ Most of our patients that are delivered at our health facility and are not discharged within 24hours after birth, but for those referred from outside hospital which present mostly with severe NNJ.

Mass media enlightenment of the population and especially pregnant women on the need to encourage them to book and have their pregnancy, delivery supervised at health facility so as to detect these conditions early and tackled them promptly in order to prevent severe hyperbilirubinaemia and its complications will go long way to reduce these problem.

\section{Conclusion}

The high rate of exchange blood transfusion in the management of severe hyperbilirubinaemia and significant mortality associated with hyperbilirubinaemia has been demonstrated in this study. The role played by lack of facility to provide qualitative and intensive phototherapy cannot be overemphasized. The provision of uninterrupted power supply and upgrading the phototherapy machines to modern fibro-optic phototherapy blanked is urgently needed. The significant contribution of late presentation to hospital, out of pocket payment of health bills has negative effect on prompt intervention. To improve on this problems of severe NNJ, there is the need for health education of the general population, especially the pregnant women on the danger of severe NNJ and the need for early booking, regular antenatal care and delivery in appropriate health facility, as well as early recognition of NNJ and prompt presentation of the affected newborn for appropriate medical intervention must be implemented in order to reduce this preventable condition.

\section{Limitation}

Our chemical pathology laboratory is not equipped to be able evaluate glucose-6-phosphate dehydrogenase enzyme deficiency which may contribute to the aetiology of neonatal jaundice. In our study, there were 12 cases of neonatal jaundice that had EBT and the causes were unclear, which might have been due to G6PD deficiency. Also, being a retrospective study and the small number of data in the study may limit the results for generalization.

Authors Contribution
All the authors conceived, wrote the draft and reviewed
the final article.
Conflict of Interest: None
Funding: None

\section{Acknowledgement}

The authors are particularly grateful to the staff of the Special Care Baby Unit and the Medical Record Department of UMTH for their support during the study.

\section{References}

1. Egri-Okwaji MTC. Exchange blood transfusion in neonatal jaundice. Niger Med J 1993;25 (5):2, 43-47

2. Ibe BC. Neonatal jaundice. In: Azubuike JC, Nkanginieme KEO, eds. Paediatric and Child Healthin a Tropical Region $2^{\text {nd }}$ ed. Owerri: African Educational Services, 2007:204-211

3. Ipek IO, Bozayakut A. Clinically significant neonatal hyperbilirubinaemia: an analysis of 546cases in Istanbul. J Trop Pediatr 2008; 54: 212-213.

4. American Academy of Pediatrics, Subcommittee on hyperbilirubinaemia. Management of hyperbilirubinaemia in the newborn infant 35 weeks or more gestation. Pediatrics 2004;114:297-316.
5. Gross S, Melhorn DK. Exchange transfusion with citrated whole blood for DIC. $J$ Paediatr 1971; 78:415-419.

6. Slusher TM, Angyo IA, BodeThomas F, McLaren DW, Wong RJ. Transcutaneous bilirubin measurements and serum total bilirubin levels in indigenous African infants. Pediatrics, 2004;113(6):16361641.

7. Olusanya BO, Akande AA, Emokpae A, Olowe SA. Infants with severe neonatal jaundice in Lagos, Nigeria. Trop Med Int Health, 2009; 14(3):277280.

8. Owa JA and Ogunlesi TA. Why we are still doing so many exchange blood transfusions for neonatal jaundice in Nigeria. World J Pediatr 2009, 5(1): 51 55
9. Onyearugha $\mathrm{CN}$, Onyire BN and Ugboma HAA. Neonatal jaundice: Prevalence and associated factors as seen in Federal Medical Centre Abakaliki, South-Eastern Nigeria. J Clin Med Res 2011;3 (3):40-45.

10. Ogunlesi TA, Ogunfowora OB. Pattern and determinants of blood transfusion in Nigerian Neonatal Unit. Niger J Clin Pract 2011;14:354-358

11. Ibekwe RC, Ibekwe MU, and Mouneke VU. Outcome of exchange blood transfusions done for neonatal jaundice in Abakaliki, South-Eastern Nigeria. J Clin Neonatal 2012;1:34-37. 
12. Thayyil S, Milligan DWA. Single versus double volume exchange transfusion in jaundiced newborn infants. Cochrane Database systematic review. 2006, issue 4.

13. Hansen TW. Extreme neonatal jaundice : How frequent is it? Acta Paediatr 2008;97:10021003.

14. MacDonald M G.; Seshia MM K.; Mullett M D.: InAveryGB (editors) Neonatology: Pathophysiology and management of the newborn: $6^{\text {th }}$ Ed. Lippincott William and wilkins Co. Philadelphia, 2005:819-835.

15. Maisels MJ, McDonagh AF. Phototherapy for neonatal jaundice. N Engl J Med 2008; 358:920-928.

16. Bahjati S, Sagheb S, Aryasepehr S, Yaghmai B. Adverse events associated with neonatal exchange blood transfusion for hyperbilirubinaemia. Indian $J$ Pediatr 2009;76:3-5.
17. Chan M. Neonatal jaundice: In Stanfield P, Brueton M, Chan M, Parkin M. (Eds) Disease of children in the tropics and subtropics ( $4^{\text {th }}$ Edition) London. Edward Arnold, pp. 221-228.

18. Mills JF, Woodgate PG. Exchange blood transfusion in neonatal jaundice (Cochrane Protocol). In: The Cochrane Data Base Systematic Reviews, Issue 3, 2003.

19. Eneh AU, Ugwu RO. Perception of neonatal jaundice amongwomen attending childrens outpatient and immunization clinic in PortHarcourt.

Niger J Clin Pract, 2009; 12(2):187-191

20. Watchko JF. Vigintiphobia revisited. Pediatrics 2005;115:1747-1753.
21. Udo JJ, Anah MU, Ochigbo SO, Etuk IS, Ekenam AD. Neonatal morbidity and mortality in Calabar, Nigeria: ahospital based study. Niger $J$ Clin Pract 2008;11(3): 285289.

22. Ezechukwu CC, Ugochukwu EF Egbounu I, Chukwuka JO. Risk factors if neonatal mortality in regional tertiary hospital in Nigeria. Niger J Clin Pract 2004; 7: 50-52.

23. Ho NK. Neonatal jaundice in Asia. Baillieres Clin Haemat. 2002;5(1): 131-142.

24. Randev S, Grover N. Predicting neonatal hyperbilirubinaemia using first day serum bilirubin levels. Indian J Pediatr 2010;77:147-150. 